\title{
Innovative adhesive film modelled on nature
}

Geckos, spiders and other insects are real climbing artists - their ability even to climb up smooth surfaces without difficulty is partly due to the van der Waals forces - but also to the special mushroom-shaped geometry of their microscopically fine adhesive hairs. A research team from Kiel has now found out why this particular shape is so important for adhesion.

n order to tackle the question as to the mechanical benefits of the mushroom shape for adhesion, an interdisciplinary research team from Kiel University (CAU) including engineering physicist Lars Heepe, biophysicist Alexander Kovalev, theoretical physicist Alexander Filippov and biologist Stanislav Gorb took a closer look at the so-called Gecko Tape - an adhesive tape developed at Kiel University in collaboration with the company Gottlieb Binder $\mathrm{GmbH}$ \& Co. KG. Its microscopic adhesive elements were inspired by the microscopically small hairs on gecko feet and leaf beetles. It adheres even to wet and slippery surfaces and can be re-used endlessly and removed without leaving any residues.

\section{Uniform stress distribution}

"To be able to study the detachment behaviour more closely, the scientists took pictures of the individual mushroomshaped microstructures at 180,000 frames per second using a scanning electron microscope. "We discovered that the actual detachment process - i.e. the period from the moment at which a defect starts to develop in the contact area to its complete separation - lasts only a few microseconds." The contact rips apart at up to $60 \%$ of the speed of sound of the material, or about 12 metres per second. "This can only be achieved if there is a uniform stress distribution between the mushroomshaped adhesive element and the surface", Heepe explains. This is the only way to store, during the detachment process, the elastic energy necessary for achieving such high speeds in such a short time.

In comparison, other adhesive geometries, such as flat punch, create stress concentrations and start to separate at the edges. With mushroom heads such as those to be found on the artificially produced Gecko Tape, a thin plate prevents the formation of stress peaks of this kind and detachment takes place from the inside to the outside. A lot of strength is necessary to do this, so adhesion is strong.

"With our experiments, we have been able to unravel an important effect of a very successful adhesion mechanism in nature", said Heepe, summing

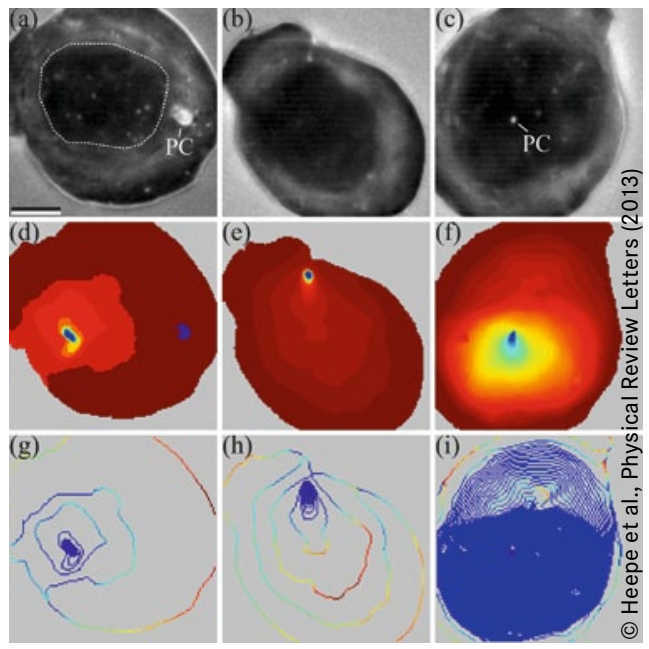

Images $\mathrm{a}, \mathrm{b}$ and $\mathrm{c}$ show three different mushroom-shaped adhesive elements of Gecko Tape in contact with glass (dark areas). Images $d$ to f show colour-coded spatiotemporal detachment maps of the adhesive elements (from a to c). Blue regions detach first and red regions detach last, whereas regions of the same colour separate from the surface at the same time. Images $g$ to i are colour-coded spatiotemporal velocity maps of local crack velocities.

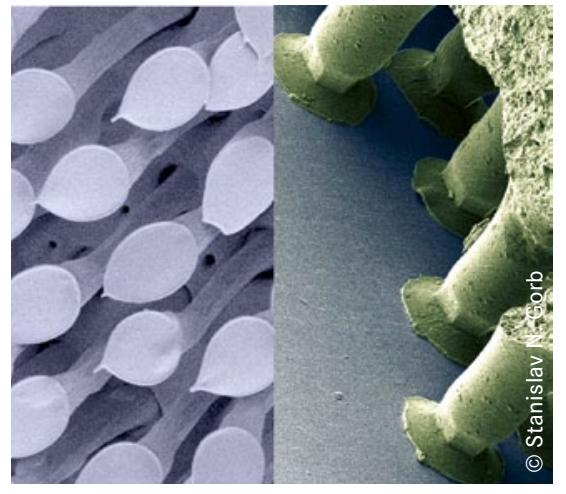

Scanning electron microscope images of the mushroom-shaped adhesive structures of a male leaf beetle (left) and of the innovative Gecko Tape (right), the adhesive elements of which are inspired by those of the beetle.

up the work of the interdisciplinary scientific team at Kiel University. Their high-speed analysis also confirms a theoretical model recently presented by an Italian group of scientists.

\section{Switchable adhesion}

The findings of the Kiel study are not only to be used as a basis for the improvement of existing adhesion structures. They are also, in the framework of the Collaborative Research Centre 677 "Function by Switching", to help to create photo-switchable adhesion systems. This suggests that it will soon be possible to develop photo-switchable adhesive systems that can be switched between an adhesive and a non-adhesive state by using light of certain wavelengths. The practical benefits of high-performance systems of this kind are obvious.".

Further information: Christian-AlbrechtsUniversität zu Kiel, Zoologisches Institut, Iheepe@zoologie.uni-kiel.de 